\title{
Short-wave infrared continuum generation in hydrogenated amorphous silicon fibers
}

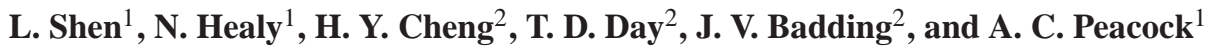 \\ 1. Optoelectronics Research Centre, University of Southampton, Southampton SO17 1BJ, United Kingdom \\ 2. Department of Chemistry and Materials Research Institute, Pennsylvania State University 16802 PA, USA \\ ls1g11@orc.soton.ac.uk
}

\begin{abstract}
Four-wave mixing and broadband continuum generation are demonstrated for the first time in a small core silicon optical fiber in the short-wave infrared wavelength regime.

OCIS codes: (060.2270) Fiber characterization; (060.2290) Fiber materials; (060.4370) Nonlinear optics.
\end{abstract}

Nonlinear silicon photonics in the short-wave infrared is currently attracting increased interest, with applications ranging from broadband telecommunications to sensing and spectroscopy. Although in this region crystalline silicon is known to have a high nonlinear figure of merit $\left(\mathrm{FOM}=n_{2} / \beta_{\mathrm{TPA}} \lambda\right)$, recent measurements conducted in our silicon optical fiber platform have shown that hydrogenated amorphous silicon (a-Si:H) can exhibit an even higher FOM owing to its consistently larger nonlinear index $n_{2}$ and even lower nonlinear absorption $\beta_{\text {TPA }}$ [1]. Compared to their on-chip counterparts, the silicon core fibers offer a number of advantages such as flexibility, robustness, cylindrical symmetry, and long waveguide lengths. To date, our nonlinear measurements have resulted in fairly modest spectral broadening, constrained by the large normal dispersion of the $\sim 6 \mu \mathrm{m}$ core fiber diameters [2]. In this paper we show that by reducing the core size to $1.7 \mu \mathrm{m}$, which shifts the zero dispersion wavelength to $\sim 2 \mu \mathrm{m}$, it is possible to generate up to $700 \mathrm{~nm}$ of spectral broadening in the short-wave infrared, similar to what has been measured in nanoscale a-Si:H waveguides on-chip [3].

The fibers are fabricated by depositing the a-Si:H material into silica capillaries with an inner diameter of $1.7 \mu \mathrm{m}$ via a high pressure chemical deposition technique. A SEM image of the fiber cross-section is shown in Fig. 1(a) together with the estimated group velocity dispersion, which indicates the fiber should exhibit anomalous dispersion when pumped beyond $2.1 \mu \mathrm{m}$. The linear loss was measured via cut-back to be $3 \mathrm{~dB} / \mathrm{cm}$ at $1.55 \mu \mathrm{m}$, decreasing to $1 \mathrm{~dB} / \mathrm{cm}$ at $2.2 \mu \mathrm{m}$, and the final fiber length was $6 \mathrm{~mm}$. To investigate spectral broadening the fiber was pumped with a Ti:Sapphire based OPO with $200 \mathrm{fs}$ pulses at $80 \mathrm{MHz}$. Fig. 1(b) shows the spectral broadening when pumped at $2.2 \mu \mathrm{m}$ in the anomalous dispersion regime. These spectra clearly show the characteristic sidebands associated with four-wave mixing which shift outwards from the pump as the power is increased, with the peak positions in good agreement with the phase-matching conditions for the fiber. Owing to the $2.4 \mu \mathrm{m}$ cut-off of the optical spectral analyser, the pump wavelength was then adjusted to $2.03 \mu \mathrm{m}$, on the edge of the normal dispersion regime, and the power increased. The spectra plotted in Fig. 1(c) show that a continuum of more than $700 \mathrm{~nm}$ can be generated over a $20 \mathrm{~dB}$ scale. In summary, by reducing our a-Si:H fiber core diameters to access the anomalous dispersion regime we have shown that it is possible to generate broadband continuum generation across the short-wave infrared region.
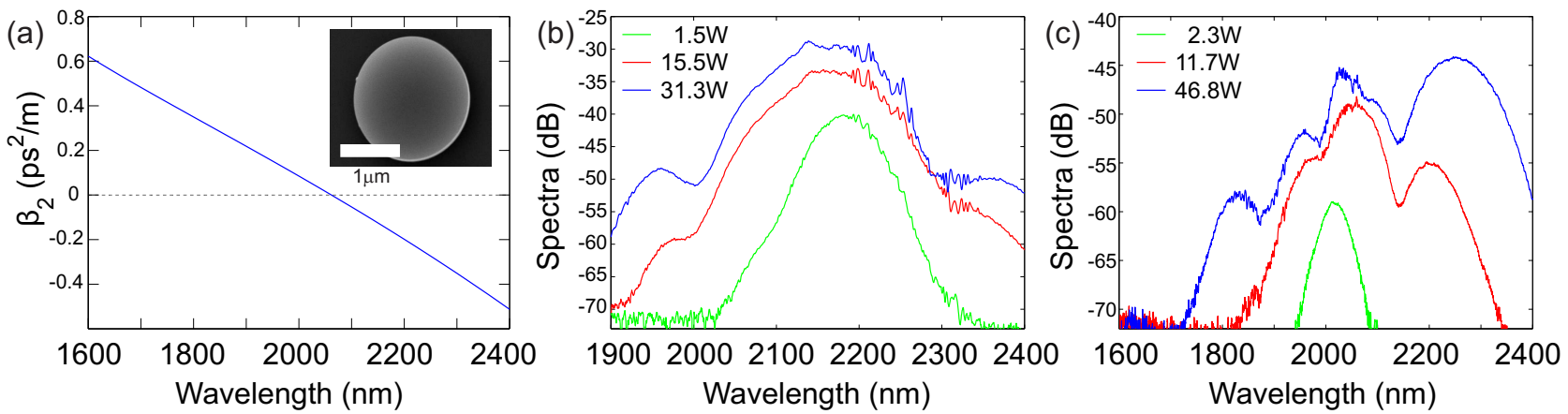

Fig. 1. (a) Simulated group velocity dispersion at short-wave infrared wavelengths; inset, SEM image of the a-Si:H core. Spectral evolutions when pumped at (b) $2.2 \mu \mathrm{m}$ and (c) $2.03 \mu \mathrm{m}$. Coupled input peak powers given in the legend.

\section{References}

1. L. Shen, N. Healy, P. Mehta, T. D. Day, J. R. Sparks, J. V. Badding, and A. C. Peacock, "Nonlinear transmission properties of hydrogenated amorphous silicon core fibers towards the mid-infrared regime," Opt. Express 21, 13075-13083 (2013).

2. P. Mehta, N. Healy, N. F. Baril, P. J. A. Sazio, J. V. Badding, and A. C. Peacock, "Nonlinear transmission properties of hydrogenated amorphous silicon core optical fibers," Opt. Express 18, 16826-16831 (2010).

3. U. D. Dave, S. Uvin, B. Kuyken, S. Selvaraja, F. Leo, and G. Roelkens, "Telecom to mid-infrared spanning supercontinuum generation in hydrogenated amorphous silicon waveguides using a Thulium doped fiber laser pump source,” Opt. Express 18, 32032-32039 (2013). 